\title{
The Biodiversity Crisis: Lessons from Phylogenetic Sagas
}

\author{
Daniel R. Brooks • Deborah A. McLennan
}

Published online: 2 September 2010

(C) Springer Science+Business Media, LLC 2010

\begin{abstract}
The future of the world's biodiversity involves preservation of individual species and, more importantly, preservation of the natural process by which the biosphere is populated. Inherited history allows species to carry within them the ability to adapt to changing circumstances. But inherited history also sets the limits for adaptation. Evolutionary potential is thus locked within shared history. Extinction removes, speciation replenishes. We must implement conservation policies that mimic the biotic expansion that sets the stage for speciation. If we do not provide space for species to spread out and find their own futures, building biodiversity reserves is tantamount to attempting to maintain standing diversity by blocking evolution. We must preserve as many species, associations, and places as possible in a geographic context large enough so that individual species may expand and contract and evolutionary dynamics can have free rein to shape the future.
\end{abstract}

Keywords Biodiversity - Conservation · Phylogenetic narrative $\cdot$ Teaching $\cdot$ Evolution $\cdot$ Historical explanations

Life sciences begin the twenty-first century with two great themes: biodiversity and biotechnology. Biotechnology, like much of post-Enlightenment science, is based on the belief that humans can understand and control their surroundings, which is why biotechnologists are often called genetic engineers. Biodiversity science, on the other

D. R. Brooks $(\bowtie) \cdot$ D. A. McLennan

Ecology and Evolutionary Biology, University of Toronto,

Toronto, ON M5S 3G5, Canada

e-mail: dan.brooks@utoronto.ca

D. A. McLennan

e-mail: deborah.mclennan@utoronto.ca hand, is based on the belief that humanity's well-being, indeed its survival, depends on understanding and accepting the limitations of fundamental biological, and hence evolutionary, principles. A wonderful example of the two different approaches can be found on television. Cut to commercial: a woman stands in her kitchen, surrounded by millions of invisible germs, her young daughter reaches for a telephone, covered in heaven knows what pathogens. Fortunately, the good mother has a chemical-laden spray that promises to kill ninety-nine percent of the germs. Daughter saved, we win. But did you ever wonder what happened to the one percent of germs that the handy spray didn't kill? Unfortunately for us, those individuals went on happily reproducing, founding a growing population immune to the germ-killing spray. Quite without knowing it, the good mother (or father) has participated in an evolutionary experiment demonstrating, yet again, the power of Darwin's principle of Natural Selection. In this case, the germ spray (or pesticide, herbicide, fungicide, antibiotic) has acted as a powerful, directional selection vector. As the old saying goes, that which does not kill you makes you stronger. In evolutionary terms, any organism that survives our concerted attempts at eradication goes on to found a stronger (more resistant) population, which requires that we invent an even more potent chemical to deal with the problem, and so the evolutionary arms race continues.

Our attempts at biotechnology have provided some stunning victories, but have also shown us that we cannot in general control or engineer nature. The biosphere is just far too complicated a system, and our knowledge of that system is far too fragmentary and simplistic. Perhaps sometime in the very (very) distant future we might "know" everything, might understand what we can and cannot change, and might have developed the maturity to accept 
those restrictions (as Dirty Harry said, “a man's gotta know his limitations"). Until then, the big question is: How do we as a species stay alive long enough to reach that future? If we can't control anything, what's the point? Let's just go down in one big global party.

\section{The Phylogenetic Narrative and Biodiversity}

While the prospect of a good party is always tempting, we believe that the situation is not grim enough to require global despair. We can begin to disentangle the complexity that is nature if we remember one vital fact: biodiversity is the product of evolution (for a summary with additional examples, see Brooks and McLennan 2002). This means that the variation characteristic of all biological populations is largely comprised of inherited history. Inherited history means that species carry within them the ability to adapt to some changing circumstances. So each time it gets colder or dryer populations don't have to sit around hoping that a mutation will occur allowing them to deal with the new problem. That's the good news. The bad news is that inherited history also sets the limits for adaptation. If you don't have the variation within your population to meet a new environmental challenge, and that challenge is critical to your survival, you go extinct. Indeed your genetic background constrains the evolutionary pathways that are available to you. If very large predators were to begin chasing horses over cliffs, no Pegasus-like creature would miraculously appear to save the species because horses simply do not have the genetic background to "make" wings and because too many other characteristics would have to change to permit powered flight. Think about the complications of changing a hoof into a wing, or reducing body size and weight enough to permit takeoff (e.g., birds have hollow bones with struts inside them for lightness and strength). This is the major difference between a Lamarckian world, in which every individual can change to meet new challenges (species do not go extinct, they just change), and a Darwinian one, in which no population has the variability necessary to meet every challenge the environment can throw at it (species go extinct because of a failure to adapt). Phylogenetic legacy is thus a doubleedged sword. When the conditions change too fast or too much, species can become "victims of [their own] history" (Wiley and Brooks 1982).

How can we use the information embodied in each species' phylogenetic narrative to try to preserve the world's biodiversity? The first thing we need to understand is the evolutionary dance between extinction (the irreversible and permanent loss of biological information as one or more species disappear) and speciation that has been going on for over three billion years now. Sometimes the two processes appear to be moving together, at others they appear decoupled, dancing on their own. Both processes are long ones, extending far beyond the lifetime (and hence research capability) of any human being, so one of the only ways we have to study them is by reconstructing their historical footprints. This is the domain of paleontology. Painstaking reconstruction of the fossil record is beginning to reveal patterns that may give us clues about underlying processes (eventually). For example, sampling the fossil record for marine vertebrate and invertebrate taxa from the Phanerozoic every ten thousand years over twenty million years revealed a series of irregular and unpredictable extinction pulses over time resembling "a forest of small events punctuated by a smaller number of high spikes." In other words, there were many intervals of a million years or more during which extinction levels were extremely low (fewer than five percent of species: coordinated stasis) interspersed with relatively few, but highly lethal, spikes during which extinction levels exceeded thirty to sixty percent of species (mass extinctions). Now the contentious part of the research begins, trying to explain what caused the irregular patterns.

Darwin (1872) argued that interspecific competition drives extinctions, with differences in fitness between species being responsible for one species completely replacing another (competitive displacement). One of the most famous examples of this process used to be the "replacement" of the South American marsupial fauna by invading North America eutherians (formerly called placental mammals) following the formation of the Isthmus of Panama. The idea was a simple one: Eutherians migrated across the newly formed isthmus into South America, where they encountered a rich marsupial fauna (confirmed by fossil evidence). Competitive interactions between invading and endemic species ensued, resulting in the eventual extinction of most marsupials, driven out of their niches by the somehow "superior" eutherians. Lessa and Fariña (1996) investigated this hypothesis by looking for a relationship between the probability of extinction in 120 mammalian orders and body mass, niche (a measure of the ecological equivalence between the invaders and the residents), place of origin (North or South America), and lineage (marsupial versus eutherian). Regression analysis revealed that only body size was linked with susceptibility to extinction (larger-bodied species were more vulnerable than smaller ones). Once the effects of body size were removed from the analysis, there was no difference in extinction rates between the two groups. In other words, there was no support for the hypothesis that marsupials died out because they were competitively inferior to eutherians. Nevertheless, the fact remains that the faunal composition did change. Lessa and Fariña suggested that the northern invaders came to dominate the southern continent because 
they had higher rates of speciation, not lower rates of extinction. At this point in the evolutionary dance, extinction and speciation were moving separately.

Erwin (1996) noted that species-poor groups of gastropods were more likely to go extinct than species-rich groups during the mid-Permian to mid-Triassic (if you are a genus with only one species, one random bad luck event can end your line, while the same event reduces your species-rich sister negligibly from 100 to 99 species). Is this a statistical artifact, or does the species-rich group possess some characteristics that are actually buffering it during an extinction period? For example, numerous authors have concluded that geographically widespread taxa appear to survive extinction events better than taxa with more limited distributions. Perhaps widespread taxa have greater dispersal abilities (and thus can move away from the area of stress to seek out a safe haven or refuge, then disperse back into available areas when the stress passes), or perhaps they are more ecologically diverse (and thus more likely to exploit a wider variety of resources, not all of which will be affected equally by the environmental stressor). It is also possible that being widespread simply decreases the likelihood that all of your populations will be unlucky enough to be in the wrong place at the wrong time.

While all of the preceding characteristics may help species survive periods of low extinction intensity (background extinction levels), it is debatable whether they have any impact during the major catastrophes. Erwin (1996) differentiated between two types of extinction: pulse (or mass) extinctions, during which the environmental stressor is so intense and rapid that species simply do not have time to adapt, and press extinctions, prolonged periods of heightened environmental stress during which species have time to respond by moving to refugia, etc. In the latter case, biological factors such as dispersal abilities, population size, body size, ecological flexibility, and so on will play a large role in determining which species survive and which do not. Surviving a press extinction does not appear to be connected with the probability of surviving a pulse extinction, emphasizing once again the importance of biological factors in the one event and the predominance of external factors and catastrophic stressors in the other.

Now let us assume that a species does manage to survive a mass extinction. What happens next? Just as we currently understand little about the forces that cause species-level extinction, we understand even less about what happens after major extinction events. The picture being developed by paleontologists indicates that some episodes of speciation and extinction follow a very general cycle: (1) major extinction event; (2) lag time, during which surviving lineages get on with the business of surviving; (3) burst of speciation in some, but not all, surviving lineages (radiations during the "recovery period"), which eventually slows down, passing into (4) a period of coordinated stasis, punctuated by random, low-level extinction spikes until the next lethal event. The time to recovery is long on a human scale. For example, reef communities, which appear to be extraordinarily susceptible to extinction events, may take five to ten million years to reassemble. Although the pattern may be a general one, it is important to realize that the timing and length of the survival and recovery periods, as well as the degree of stability during the period of stasis, varies across ecosystems and localities, and through time. In one way or another, however, life always comes surging back. Given this, the central questions that must be addressed by current and future generations of researchers are: What causes the surge? Why do some, but not all, groups participate in the radiation? And is there any evidence that groups which have participated in one radiation will do so again in the future (once a speciesrich group, always a species-rich group, and vice versa)?

Of course, not all studies of speciation and extinction involve hundreds of millions of years and fossils. For example, historical biogeographers study speciation by examining the relationship between phylogenetic (species relationships) and geological patterns (e.g., the timing of mountain upheavals, the birth of islands, the formation of an isthmus). Phylogenetic studies of the evolution of mainland and island archipelago biotas have found alternating nodes of expansion, where lineages are produced between regions, and isolation, where lineages are produced within a region (e.g., Bouchard et al. 2004; Brooks and Folinsbee 2005; Halas et al. 2005; Brooks and Hoberg 2007; Lim 2008; Eckstut et al. 2010). According to this dynamic, dubbed the taxon pulse (Erwin 1981), biodiversity is produced by alternating waves of geographic isolation (with in situ speciation: speciation that occurs at the site of a lineage's origin), punctuated by episodes of colonization into available areas. The episodes of expansion are highly correlated with episodes of regional and global climate change, so the taxon pulse may represent a general response to climate change, including large-scale extinctions. In Darwinian terms, species move to a new location where the old conditions still persist, or they go extinct. Rarely are species able to stay in place and adapt to the new conditions.

\section{Support, Don't Hinder, Evolution}

So what do we know? Extinction, like death, is a fact of life. Evolutionary diversification, like birth, is the means by which the biosphere renews itself following each extinction event. All biodiversity and conservation efforts must thus be aimed toward preserving as many elements of the biosphere as possible under conditions that will allow them to continue evolving; that is the only way the biosphere can 
continue to replenish itself in the face of inevitable "natural" and anthropogenic extinctions.

The most persistent units of biodiversity are species, which are vessels of future potential as well as living legacies of past modifications and stasis, shaped by millennia of biotic and abiotic interactions partitioned into ecologically coherent units. They are history-embodied. The predictable parts of biological systems are the stable elements, form and function, autecological and synecological, which have persisted through evolutionary time. Shared history allows us to make predictions, and this saves time and money, two resources that are in short supply in battling the biodiversity crisis.

Just what do we mean by "the predictive power of shared history"? Two examples should help explain this idea. One of the most significant recent contributions to breast cancer therapy was the discovery of a substance called Taxol produced by a tree species called the American yew. If we wanted to search for additional sources of Taxol, the most obvious place to look would be the sister species of the American yew, the European yew, because sister species are likely to share many traits in common. Sure enough, the European yew does produce a variant of Taxol, called Taxotene, which also has anti-cancer properties. Using the phylogenetic narrative has thus allowed us to efficiently identify a new species that is useful to humanity, which is a win-win situation because it decreases the "cost of doing business" while increasing the probability that the new species will be preserved.

Now consider the boulder darter (Etheostoma wapiti), a small fish inhabiting fast flowing riffles of streams in southern Tennessee and northern Alabama. Until recently, little was known of its breeding behavior, which was problematic given that the boulder darter was federally listed as endangered. Fortunately, we did know that E. wapiti is a member of a clade comprising six species, five of which display the derived character of depositing their eggs beneath or behind large slab rocks. Based on that information, and taking into account that $E$. wapiti is also found in habitats containing slab rocks just like its relatives, we would predict that it would have the same breeding strategy as its relatives; experimental studies confirmed that prediction. Understanding the breeding system, in turn, allowed researchers to determine that the boulder darter is endangered because hydroelectric dams upstream cause fluctuating water levels. Drops in those levels during the breeding season expose the boulders and the underlying egg masses, killing the next generation of darters. If more information about the behavior and ecology of the boulder darter is required to formulate conservation policies, studies could be conducted using its widespread and relatively common sister species, Etheostoma vulneratum, without disturbing the endangered populations. Obviously, predict- ing the requirements of endangered or rare species based upon information from close relatives is not foolproof because each species has its own suite of unique characteristics. It does, however, provide a starting point for studies based on information other than inspired guesswork and thus buy us time in the race against species-level extinction.

But what should we do in the event of a large-scale or mass extinction? Can we tell which species are likely to produce more species and which are likely to be evolutionary dead ends? Here, paleontological and phylogenetic studies agree that there appear to be few, if any, global generalizations about biodiversity, so the best choice may depend on the clade being assessed. As a consequence, it is important to understand the biological needs of as many species as possible, so we can make a decision about the allocation of time and resources for their preservation. More importantly, we must remember that species do not arise or live in isolation. Rather, they evolve in, and are parts of complex ecosystems occurring in particular places (and times) on this planet. But because each species carries with it a substantial phylogenetic legacy, the ecosystems in which they reside are repositories of enormous amounts of evolutionary history. So, do we protect old ecosystems, full of old species or young ecosystems full of young species? Do we preserve simple ecosystems or more complex ones? Or preserve some mixture?

Historical biogeography identifies areas of endemism that have been hot spots of evolutionary activity in the past. Such areas do not always encompass the greatest number of extant species, nor are they always extremely large or centrally located. For example, a species-rich area may represent a region of overlap between two biotas. Such an area may represent marginal habitat that has limited the expansion of both biotas in the past. Since closely related species tend to utilize the same resources, preserving regions of overlap, to the exclusion of the larger areas that contain most of the preferred habitat for the species in each biota, could be counterproductive for the survival of any of them. Such forced competition could lead to a rapidly cascading series of regional extinctions, the exact opposite of our intention. Additionally, if the taxon pulse is the general dynamic for producing biodiversity over long periods of time, what kind of conservation measures do we need to undertake to preserve the potential for future pulses? The initial design criteria for biodiversity reserves were based on the MacArthur-Wilson model of island biogeography. Within a decade, workers were divided into two camps, the single large (SL) or (O) several small (SS) reserve camps. Both sides of the SLOSS debate have missed an important point: biological diversity is evolved diversity, and evolution does not occur within humandefined spatial boundaries, large or small. Preserving biological diversity requires conserved areas as well as 
connections ("dispersal corridors") between them permitting biotic expansion (and the cycle of taxon pulses), setting the stage for the production of new species and ecological associations.

Shared phylogenetic history imparts an additional evolutionary element to ecosystems. Ecosystems in areas of endemism contain many species that have been associated with each other for a long time. The stability and resilience of any given ecosystem in the face of perturbations (including anthropogenic ones) stem from a core of old species and old interactions. This phylogenetic conservatism may also make ecosystems slow to respond to drastic environmental changes. Our ability to predict the behavior of a community under a variety of disturbances thus depends less on the overall diversity of the ecosystem than on our understanding of the community's evolutionary legacy. The longer species have been associated with each other, the greater the chances that they have become co-adapted, and dependent upon each other, to a greater or lesser degree. This means some species may be more important than others for maintaining ecosystem integrity, even if they are not obvious repositories of special genetic significance. For example, each mass extinction spike for marine Phanerozoic species was closely preceded by a smaller, yet significant, spike. The initial spikes might be species whose extinction catalyzed ecological cascade effects, enhancing the subsequent mass extinction.

\section{Conclusions}

Evolutionary potential is locked within shared history, the outcome of a three-billion-year give and take between extinction and speciation, the yin and yan of biology. Extinction removes, speciation replenishes (albeit not with the same things as before). We must therefore implement conservation policies that mimic, insofar as possible, the biotic expansion that sets the stage for speciation. If we do not provide space for species to spread out and find their own futures, building biodiversity reserves is tantamount to creating zoos and herbaria in a desperate attempt to maintain standing diversity by blocking evolution. No matter how well intentioned, this approach is ultimately doomed because extinction will happen when you are trying to micromanage millions of species. Ultimately then, we must preserve as many species, associations, and places as possible in a geographic context large enough so that individual species may expand and contract and evolutionary dynamics can have free rein to shape the future. We cannot control the future, but we can do our best to protect the integrity of the one process by which biodiversity can be renewed-evolution.

Acknowledgments Our thanks to Niles Eldredge for conceiving this special issue, and to the authors who have contributed to it.

\section{References}

Bouchard P, Brooks DR, Yeates DK. Mosaic macroevolution in Australian wet tropics arthropods: community assemblage by taxon pulses. In: Moritz C, Bermingham E, editors. Rainforest: past, present, future. Chicago: University of Chicago Press; 2004. p. $425-69$.

Brooks DR, Folinsbee KE. Paleobiogeography: documenting the ebb and flow of evolutionary diversification. In: Lieberman BS, Stigall Rode A, editors. Paleontological society papers. 2005; 11: $15-43$.

Brooks DR, Hoberg EP. Darwin's necessary misfit and the sloshing bucket: the evolutionary biology of emerging infectious diseases. Evolution: Education and Outreach. 2007;1:2-9.

Brooks DR, McLennan DA. The nature of diversity: an evolutionary voyage of discovery. Chicago: University of Chicago Press; 2002.

Darwin C. The origin of species. 6th ed. London: John Murray; 1872. Eckstut ME, McMahan CD, Crother BI, Ancheta JM, McLennan DA, Brooks DR. PACT in practice: comparative historical biogeographic patterns and species-area relationships of the Greater Antillian and Windward Hawaiian Island Terrestrial Biotas. Global Ecology and Biogeography. 2010.

Erwin DH. Understanding biotic recoveries: extinction, survival, and preservation during the end-Permian mass extinction. In: Jablonski D, Erwin DH, Lipps JH, editors. Evolutionary paleobiology. Chicago: University of Chicago Press; 1996. p. 398-418.

Erwin TC. Taxon pulses, vicariance, and dispersal: an evolutionary synthesis illustrated by carabid beetles. In: Nelson G, Rosen DE, editors. Vicariance biogeography: a critique. New York: Columbia University Press; 1981. p. 159-96.

Halas D, Zamparo D, Brooks DR. A protocol for studying biotic diversification by taxon pulses. J Biogeogr. 2005;32:249-60.

Lessa EP, Fariña RA. Reassessment of extinction patterns among the Late Pleistocene mammals of South America. Palaeontology. 1996;39:651-62.

Lim BK. Historical biogeography of New World emballonuird bats (tribe Diclidurini): taxon pulse diversification. J Biogeogr. 2008;35:1385-401.

Wiley EO, Brooks DR. Victims of history-a nonequilibrium approach to evolution. Syst Zool. 1982;31:1-24. 